ducibly connected between $A$ and $B$, and hence satisfies all the conditions of Lennes'* definition of a simple continuous arc from $A$ to $B$.

The chief interest in the above definition of a simple continuous arc lies in the fact that in its statement nothing is said concerning the closure or the boundedness of the set in question. This result for the case of the arc is related to results obtained by R. L. Wilder $†$ for the case of a simple closed curve. Wilder found that definitions of a simple closed curve could be given in which the closed and bounded conditions were replaced, or modified, by adding the condition of regularity.

The University of TeXas

\title{
NOTE ON THE FOURIER DEVELOPMENT OF CONTINUOUS FUNCTIONS $\ddagger$
}

\author{
BY A. H. COPELAND
}

If we have given a function, $f(t)$, which is continuous and periodic, this function is not necessarily developable in a Fourier series, but by a monotone change of variable it becomes so. We shall prove in fact the following theorem.

Theorem. If a function, $f(t)$, is continuous and periodic with period $b-a$, then there exists a monotone continuous function, $t=t(\theta)$, transforming the interval $(0,2 \pi)$ into the interval

* N. J. Lennes, loc. cit. Hallett has shown that the condition of boundedness in Lennes' definition is superfluous. See G. H. Hallett, Jr., Concerning the definition of a simple continuous arc, this Bulletin, vol. 25 (1919), pp. 325-326. The same result was published two years later by Knaster and Kuratowski in their paper Sur les ensembles connexes, loc. cit., p. 224, Theorem 27.

$\dagger$ See an abstract of a paper On the definition of simple closed curve, this Bulletin, vol. 32 (1926), p. 123. See also p. 591.

$\ddagger$ Presented to the Society, April 16, 1927. 
$(a, b)$, such that $f[t(\theta)]=F(\theta)$ is developable in a uniformly convergent Fourier series in the interval $(0,2 \pi) .^{*}$

Such a transformation does not alter the general character of the function $f(t)$.

The proof of this theorem depends upon a theorem of Fejér $\dagger$ to the effect that if a power series converges to a function $w(z)$ within the unit circle $|z|=1$, and if $w(z)$ maps conformally the interior of the unit circle onto the interior of a region bounded by a closed Jordan curve, then the power series converges uniformly on the boundary of that circle.

We shall also have need of the following lemma.

Lemma. Given a function $\phi(t)$ which is continuous for $a \leqq t \leqq b$, and such that $\phi(a)=\phi(b)=0$, then there exists a continuous function, $\sigma(t)$, which is of limited variation and such that $\sigma(a)=\sigma(b)=0$ and $\phi(t)-\sigma(t) \geqq 0$ when $a<t<b$.

Let $m$ be the minimum of $\phi(t)$ in the interval $(a, b)$. If $m \geqq 0$ we can let $\sigma(t)=0$. Let us, then, consider the case $m<0$. Let $\alpha$ and $\beta$, respectively, be the lower and the upper bounds of the set of roots of the equation $\phi(t)=m$. Let $\sigma(t)$ be the minimum of $\phi(t)$ in the interval $(a, t)$ when $a \leqq t \leqq \alpha$, let $\sigma(t)=m$ when $\alpha<t<\beta$, and let $\sigma(t)$ be the minimum of $\phi(t)$ in the interval $(t, b)$ when $\beta \leqq t \leqq b$. Then $\sigma(t)$ is seen to be continuous and to satisfy the conditions $\sigma(a)=\sigma(b)=0$ and $\phi(t)-\sigma(t) \geqq 0$ for $a \leqq t \leqq b$. Moreover, $\sigma(t)$ is monotone in the intervals $(a, \alpha)$ and $\beta, b)$; and hence it is of limited variation in the interval $(a, b)$.

If, instead of the function $\sigma(t)$, we take the function $\rho(t)=\sigma(t)-\sin [\pi(t-a) /(b-a)]$, then $\phi(t)-\rho(t)>0$ when $a<t<b$. Furthermore $\rho(t)$ is continuous and of limited variation, and $\rho(a)=\rho(b)=0$.

* Suggested by G. C. Evans, The Logarithmic Potential, Colloquium Series, American Mathematical Society (in press). Chap. VII.

$\dagger$ See L. Fejér, Über die Konvergenz der Potenzreihe an der Konvergenzgrenze in Fällen der konformen Abbildung auf die schlichte Ebene, Mathematische Abhandlungen Hermann Amandus Schwarz zu seinem fünfzigjährigen Doktorjubiläum gewidmet. 
Let us consider the function $f(t)$. Without loss of generality we may assume that $f(0)=f(2 p)=0$, where $2 p$ is the period of this function. Let

where

$$
\phi(t)=f(t)-\frac{f(p)}{p} v(t),
$$

$$
\begin{array}{ll}
v(t)=t, \quad \text { if } \quad 0 \leqq t \leqq p, \\
v(t)=2 p-t, \text { if } \quad p \leqq t \leqq 2 p .
\end{array}
$$

Then $\phi(0)=\phi(p)=\phi(2 p)=0$. It follows from the preceding lemma that there exists a function $\rho(t)$ which is of limited variation, continuous, and such that $u(t)=\phi(t)-\rho(t)$ is zero when $t=0, p$, or $2 p$, and $u(t)$ is otherwise positive in the interval $(0,2 p)$.

Next let $u(t)=u_{1}(t)+u_{2}(t)$, where

$$
\begin{aligned}
& \left\{\begin{array}{lll}
u_{1}(t)=u(t), & \text { if } \quad 0 \leqq t \leqq p, \\
u_{1}(t)=0, & \text { if } \quad p \leqq t \leqq 2 p
\end{array}\right. \\
& \left\{\begin{array}{lll}
u_{2}(t)=0, & \text { if } \quad 0 \leqq t \leqq p, \\
u_{2}(t)=u(t), & \text { if } \quad p \leqq t \leqq 2 p .
\end{array}\right.
\end{aligned}
$$

The pairs of functions $\left[u_{1}(t), v(t)\right]$ and $\left[u_{2}(t), v(t)\right]$ define two closed Jordan curves, $C_{1}$ and $C_{2}$.

The region interior to $C_{1}$ can be mapped conformally onto the interior of the unit circle, $|z|=1$. Between the points of $C_{1}$ and the points of the unit circle, there is a one-to-one and continuous correspondence preserving cyclic order. ${ }^{*}$ That is, there exists a continuous monotone function, $t=t_{1}(\theta)$, relating the points, $z=e^{i \theta}$, of the unit circle to the points, $w=u_{1}(t)+i v(t)$, of the curve $C_{1}$. In particular, the mapping may be accomplished in such a manner that the points $\theta=0$, $\theta=\pi$ correspond respectively to the points $t=0, t=p$. Then $t_{1}(0)=0$ and $t_{1}(\pi)=p$.

* See C. Carathéodory, Über die gegenseitige Beziehung der Ränder bei der konformen Abbildung des Inneren einer Jordanschen Kurve auf einen Kreis, Mathematische Annalen, vol. 73 (1913), pp. 305-320. 
Let $w=w_{1}(z)$ be the mapping function. Then $w_{1}(z)$ can be expanded in a power series about the origin. This series converges when $|z|<1$. Furthermore, by the theorem of Fejer, this series converges uniformly on the boundary of the circle $|z|=1$. It follows that the function $U_{1}(\theta)=u_{1}\left[t_{1}(\theta)\right]$ is developable in a uniformly convergent Fourier series.

In a like manner it can be seen that there exists a function $t=t_{2}(\theta)$ which is monotone, continuous, and such that $t_{2}(0)=0$ and $t_{2}(\pi)=p$, and also such that the function $U_{2}(\theta)=u_{2}\left[t_{2}(\theta)\right]$ is developable in a uniformly convergent Fourier series.

The sum $U_{1}(\theta)+U_{2}(\theta)$ defines a function $U(\theta)$ which is developable in a uniformly convergent Fourier series in the interval $(0,2 \pi)$, and we shall show that there exists a monotone continuous function $\theta=\theta(t)$ such that $U[\theta(t)] \equiv u(t)$. To do this, let us define a function $t(\theta)$ by the equations

$$
\begin{cases}t(\theta)=t_{1}(\theta), & \text { if } \quad 0 \leqq \theta \leqq \pi, \\ t(\theta)=t_{2}(\theta), & \text { if } \quad \pi \leqq \theta \leqq 2 \pi,\end{cases}
$$

and let the inverse of $t=t(\theta)$ be $\theta=\theta(t)$. Then these two functions are continuous and monotone. Moreover

$$
\begin{aligned}
& U_{1}[\theta(t)]+U_{2}[\theta(t)]=u_{1}(t), \text { when } 0 \leqq t \leqq p, \\
& U_{1}[\theta(t)]+U_{2}[\theta(t)]=u_{2}(t), \text { when } p \leqq t \leqq 2 p,
\end{aligned}
$$

since $U_{1}(\theta)=0$ in the interval $(\pi, 2 \pi)$, and $U_{2}(\theta)=0$ in the interval $(0, \pi)$. Thus $U_{1}[\theta(t)]+U_{2}[\theta(t)] \equiv u(t)$. Furthermore the functions $v[t(\theta)]$ and $\rho[t(\theta)]$ are of limited variation with respect to $\theta$. Therefore

$$
F(\theta)=U_{1}(\theta)+U_{2}(\theta)+\rho[t(\theta)]+\frac{f(p)}{p} v[t(\theta)]
$$

is developable in a uniformly convergent Fourier series. But $F(\theta)=f[t(\theta)]$; hence the theorem is established.

The Rice Institute 